\title{
Delivering Quality Healthcare in India: Beginning of Improvement Journey
}

\author{
ASHOK DeORARI ${ }^{1}$ AND Nigel LiVESLEY ${ }^{2}$ \\ ${ }^{1}$ WHO Collaborating Centre for Education \& Research in Newborn Care, Department of Pediatrics, AIIMS, New Delhi, India; and \\ ${ }^{2}$ USAID-ASSIST Project, University Research Co., MD, USA. ${ }^{1}$ ashokdeorari_56@hotmail.com
}

I mproving the quality of healthcare, especially in the low- and middle-income countries is essential to meet the health-related targets of the Sustainable Development Goals (SDG). This is especially true for improving maternal, fetal, neonatal and child survival as recognized in the 'Every Newborn Action Plan (ENAP)' and its adaptation by India and the constituent states. This special issue of Indian Pediatrics highlights mechanisms that can support healthcare providers in using various quality improvement (QI) approaches and showcases some of the improvement projects undertaken in the country by different teams.

Quality healthcare is safe, effective, patient-centered, timely, efficient, and equitable. Optimal resources and clinical skills are clearly necessary to deliver quality care, but are not enough by themselves [1]. Approaches to ensure that patients receive high quality care can be divided into two general categories: quality assurance (QA) and quality improvement (QI). QA focusses on ensuring that requisite infrastructure, supplies and trained staff are in place to facilitate delivery of quality care. It relies on periodic audits (typically by external evaluators) to determine whether predefined standards are being met and thus identifies gaps that need to be addressed. QA is typically the responsibility of health system administrators. QI approach, on the other hand, focus on equipping front-line healthcare workers and managers with skills to identify and solve problems at their level. QA and QI are two sides of same coin, and both are essential to ensure optimal functioning of health system. QI approaches have been less widely used in healthcare till recently, but provide health workers and health system administrators with tools that can help solve quality problems that QA approaches are not able to address.

The QI approaches focus on narrowing the gap between current knowledge and actual practices in the real world. They share four core principles. First, working in multi-disciplinary teams, which include clinical experts who know what should be happening to patients as well as front-line staff delivering the care who know what is actually happening to patients. Second, attention to understanding local systems to identify barriers preventing delivery of quality care, from which potential solutions emerge. Third, testing the potential solutions using sequential small tests of change (Plan-DoStudy-Act (PDSA) cycles) to learn if they are feasible and effective, and to adapt them to the local context. Fourth, a focus on using measurement and data to understand problems, and learn if solutions are achieving their objectives.

This journal issue contains papers that $(i)$ illustrate the cardinal principles of QI implementation; ( $i$ i) provide examples of how this approach has been used to address the six dimensions of quality in the Indian context; and (iii) describe some resources that can support health workers in using the QI tools.

\section{LESSONS FROM QI IMPLEMENTATION}

Leadership plays a crucial role in imbibing and sustaining QI. An effective leader would have enough leverage to remove the obstacles to improvement in the specific target area. Additionally, there is a need for local champions who drive the QI projects on day-to-day basis. The enthusiasm and momentum that these 'project champions' bring to the team is crucial. The team must include all stakeholders who have knowledge of the key processes involved. Otherwise it may result in wrong choice of problem to be addressed, faulty analyses and flawed improvement approaches. Sivanandan, et al. [2] report how all the above ingredients can be integrated in creating a culture of QI that enables running and sustaining multiple such projects in a unit.

Despite knowing what care should be provided, implementation in local context of a healthcare facility is a challenge to be surmounted by committed teams and local champions. The science of QI is the missing link that can bridge the gap between the prevalent and the desirable. The studies by Maria, et al. [3] and Dudeja, 
et al. [4] demonstrate this translation of standard guidelines for babies born by cesarean section into practice in their respective hospitals using PDSA cycles for continuous QI.

Measurement is critical for improvement. Data collection and analysis for QI projects requires clear operational definitions and rigor to ensure validity. Measurements that are few but focused on providing direction to the processes of change are the sine qua non of data handling for QI projects. Chawla and Darlow [5] in their article describe the role of data in a quality monitoring and improvement system in a simplified way.

\section{Examples of QI Methods Addressing the SiX Dimensions OF QUALITY}

Very common and important issues like healthcareassociated infections, including catheter-related blood stream infections, continue to remain seminal safety issues in neonatal intensive care units (NICU), and these cannot be solved by a blanket solution at the level of policy makers. The solutions must come from the ground level. This is emphasized in the articles by Balla, et al. [6] and Khurana, et al. [7] who have demonstrated the use of QI methods to decrease infection-related morbidity and optimize resource utilization.

An important facet of improvement process is to find newer ideas for problem solving. Going beyond the routine care provided by the healthcare staff, the involvement of the family in the NICU has been thought to be critical for optimizing physical and developmental outcomes of preterm and sick neonates. Joshi, et al. [8] worked with family members to increase the duration of Kangaroo mother care (KMC) in low birth weight (LBW) babies while Thakur, et al. [9] improved the usage of expressed breast milk (EBM) in the admitted very low birth weight (VLBW) babies by involving mothers in the QI efforts.

In developing countries, patient load has been considered as the biggest hurdle to delivering quality care, especially in public hospitals. Quality science looks at opportunities for improvement even within the constrained resources and improving patient experience by bringing efficiency in the health system. Chandra and colleagues $[10,11]$ in their articles demonstrate how waiting times in operation theater and for Retinopathy of Prematurity (ROP) screening can be reduced by testing and implementing new ideas even in a busy public hospital. In another article, Mallick, et al. [12] report a successful QI project that reduced the time for triaging. Such efforts are bound to improve the outcomes of sick neonates.

\section{Mechanisms That can Support Health Workers TO USE QI METHODS}

There is an abundance of evidence-based guidelines and recommendations postulating the ideal healthcare standards. The World Health Organization (WHO) has laid out the standards of care for maternal and newborn care [13], and for care of children and young adolescents [14]. Such standards help QI teams identify areas for improvement.

Innovative solutions are needed to reach interiors of India to educate health professionals in QI methodology and creating a learning sharing platform. Mehta, et al. [15] describe a learning platform for the South-East Asia Region of WHO for collaborative learning and use of digital technology to reach healthcare provider via virtual e-Learning (www.pocqi.org) and smartphone applications.

Variations in clinical outcomes across different healthcare facilities that cannot be explained by differences in the patient-mix are well reported. Differences in care practices contribute to this variation and provide an opportunity for learning and improvement. Collaborative networking, especially in perinatal and neonatal care, is still in a nascent stage in India. Murki, et al. [16] describe how collaborative networking can help improve outcomes and what challenges need to be surmounted for establishing such networks.

For reducing maternal, newborn and child mortality, the focus has been on reaching higher coverage with key effective interventions. Often, these evidence-based interventions are delivered with insufficient quality [16]. Poor quality of care may even be harmful for the health of the individual by causing iatrogenic harm - 'doing too much or too little' is bad in healthcare [17]. Patient safety, use of checklist and avoiding medication errors are paramount for improving health outcomes [18]. Quality healthcare and patient safety need to be in curriculum of future graduate students as poor experience in hospitals threatens future health-seeking behavior of the families and communities. Low utilization of healthcare services by the population and lack of adequate progress towards achieving Millennium Development Goal 4 and 5 has been partially attributed to the poor quality of the services [19]. Quality of care is embedded in the recently developed global frameworks like ENAP and Ending preventable maternal mortality (EPMM) [20]. It is therefore mandatory that interventions are delivered with sufficient quality - meeting appropriate standards of care. 
Funding: This special issue of the journal was made possible by the support of the American people through the United States Agency for International Development (USAID), through the USAID Applying Science to Strengthen and Improve Systems (ASSIST) Project, implemented by University Research Co., LLC under Cooperative Agreement Number AID-OAA-A-1200101.

Competing interest: None stated.

\section{REFERENCES}

1. Das J, Woskie L, Rajbhandari R, Abbasi K, Jha A. Rethinking assumptions about delivery of healthcare: implications for universal health coverage. BMJ. 2018;361:k1716.

2. Sivanandan S, Sethi A, Joshi M, Thukral A, Sankar MJ, Deorari AK, et al. Gains from quality improvement initiatives - experience from a tertiary-care institute in India. Indian Pediatr. 2018;55:809-17.

3. Maria A, Shukla A, Wadhwa R, Kaur B, Sarkar B, Kaur M. Achieving early mother-baby skin-to-skin contact in caesarean section: A quality improvement initiative. Indian Pediatr. 2018;55:765-7.

4. Dudeja S, Sikka P, Jain K, Suri V, Kumar P. Improving first-hour breastfeeding initiation rate after cesarian deliveries: A quality improvement study. Indian Pediatr. 2018;55:761-4.

5. Chawla D, Darlow BA. Development of quality measures in perinatal care - priority for developing countries. Indian Pediatr. 2018;55:797-802.

6. Balla KC, Rao SPN, Arul C, Shashidhar A, Prashantha YN, Nagaraj S, et al. Decreasing central line-associated bloodstream infections through quality improvement initiative. Indian Pediatr. 2018;55:753-6.

7. Khurana S, Saini SS, Sundaram V, Dutta S, Kumar P. Reducing healthcare-associated infections in neonates by standardizing and improving compliance to aseptic nontouch techniques: A quality improvement approach. Indian Pediatr. 2018;55:748-52.

8. Joshi M, Sahoo T, Thukral A, Joshi P, Sethi A, Agarwal $\mathrm{R}$. Improving duration of kangaroo mother care in a tertiary-care neonatal unit: A quality improvement Initiative. Indian Pediatr. 2018;55:744-7.

9. Thakur A, Kler N, Garg P, Singh A, Gandhi P. Impact of quality improvement program on expressed breastmilk usage in very low birth weight infants. Indian Pediatr. 2018;55:739-43.
10. Chandra P, Tewari R, Dolma Y, Das D, Kumawat D. Reducing preoperative waiting-time in a pediatric eye operation theater by optimizing process flow: A pilot quality improvement project. Indian Pediatr. 2018;55:773-5.

11. Chandra P, Kumawat D, Tewari R, Panyala RR, Sreeshankar SS. Reducing waiting-time of preterm babies at retinopathy of prematurity clinic: A quality improvement project. Indian Pediatr. 2018;55:776-9.

12. Mallick A, Banerjee M, Mondal B, Mandal S, Acharya B, Basu B. A quality improvement initiative for early initiation of emergency management for sick neonates. Indian Pediatr. 2018;55:768-72.

13. WHO: Standards for Improving Quality of Care for Maternal and Newborn Care in Health Facilities. Available from: http://www.who.int/maternal_child_adolescent/ documents/improving-maternal-newborn-care-quality/en. Accessed August 07, 2018.

14. WHO. Standards for Improving the Quality of Care for Children and Young Adolescents in Health Facilities. Available from: http://origin.who.int/maternal_child_ adolescent/documents/quality-standards-child-adoles cent/en/. Accessed August 07, 2018.

15. Mehta R, Sharma KA. Use of learning platforms for quality improvement. Indian Pediatr. 2018;55:803-8.

16. Murki S, Kiran S, Kumar P, Chawla D, Thukral A. Quality improvement collaborative for preterm infants in healthcare facilities. Indian Pediatr. 2018;55:818-23.

17. Souza JP, Gulmezoglu AM, Vogel J, Caroli G, Lumbiganoo P, Qureshi Z, et al. Moving beyond essential interventions for reduction of maternal mortality (the WHO Multicountry Survey on Maternal Newborn Health): Cross sectional study. Lancet. 2013;18:381 (9879):1747-55.

18. Dutta S, Raghuveer T, Vinekar A, Dogra MR. Can we stop the current epidemic of blindness from retinopathy of prematurity. Indian Pediatr. 2016;53(suppl.2):S80-4.

19. World Health Organization. Patient Safety: Making Health Care Safer. Available from: http://www.who.int/ patientsafety/publications/patient-safety-making-healthcare-safer/en/. Accessed August 07, 2018.

20. World Health Organization. Strategies Toward Ending Preventable Maternal Mortality (EPMM). Geneva: World Health Organization, 2015. Available from: http:// who.int/reproductivehealth/topics/maternal_perinatal/ epmm/en/. Accessed August 07, 2018. 